\title{
Fermi Liquid Theory for the Persistent Current Past a Side-Coupled Quantum Dot
}

\author{
Ian Affleck \\ Department of Physics and Astronomy, University of British Columbia, Vancouver, British Columbia, Canada V6T 1Z1 \\ Erik S. Sørensen \\ Department of Physics and Astronomy, McMaster University, Hamilton, ON, L8S 4 M1 Canada
}

(Dated: July 21, 2017)

\begin{abstract}
A Fermi Liquid theory is developed for the persistent current past a side coupled quantum dot yielding analytical predictions for the behavior of the first two harmonics of the persistent current as a function of applied magnetic flux. The quantum dot is assumed weakly coupled to a ring of non-interacting electrons and thus appropriately described as a Kondo impurity. The theory is valid at weak Kondo couplings in the regime where the system size, $L$, is much larger than the size of the Kondo screening cloud, $\xi_{K}$. The predictions of the Fermi Liquid theory are compared to exact diagonalization results for the persistent current that lend support to the existence of a regime correctly described by this theory. The finite temperature conductance, at $T \ll T_{K}$ is also calculated using Fermi liquid theory allowing the definition of a "Wilson ratio" relating the conductance and the persistent current.
\end{abstract}

PACS numbers: 72.10.Fk, 73.23.Ra, 72.15.Qm, 73.23.-b

\section{INTRODUCTION}

The recent experimental observation of the Kondo effect and related physical phenomena in quantum dots $, 1,2,3,4$ quantum corrals ${ }^{5}$ molecular electronics devices $\frac{6.7}{7}$ and carbon nanotubes $\frac{8}{-}$ as well as related nanostructures has led to significant renewed interest in this fundamental effect. For a review of recent progress see Ref. 9. The Kondo effect in ordinary metals is usually associated with an increase in the resistance at low temperatures but experiments performed on these nanostructures $1.4,5,8$ have shown that the Kondo effect can also lead to an increase in the conductance that in some settings can reach the unitary limit $\left(G=2 e^{2} / h\right)$, completely overcoming the Coulomb blockade. The fundamental and technological interest in the complete understanding of this phenomenon is therefore considerable. The experiments are typically performed on semiconductor quantum dots where the electron occupation number on the dot is controllable by a gate voltage. When the occupation number is odd the quantum dot can act as a spin $s=1 / 2$ impurity screened by the electrons in the leads. The screening of the impurity spin by the conduction electrons is, in some circumstances, associated with the formation of a "screening cloud" of size $\xi_{K}=v_{F} / T_{K}$ surrounding the impurity, 10,11,12,13,14,15,16 Here $v_{F}$ is the Fermi velocity and $T_{K}$ the Kondo temperature.

We note that the picture of a screening cloud of size $v_{F} / \xi_{K}$ is valid in a one dimensional system and also in an infinite two or three dimensional system where only one channel (such as the s-wave) couples most strongly to the Kondo impurity. However the behavior may be rather different in a disordered two or three dimensional box in which all states couple strongly to the Kondo impurity. For the 3-dimensional model considered in [17], for instance, the effective screening cloud is much smaller, $\propto T_{K}^{-1 / 3}$. See also [18].

The experimental observation of the screening cloud has proven elusive but recent experiments $\underline{4}$ have suggested that it might be observable in finite-size properties of the conductance when the dot is attached to a single quantum wire that forms an Aharonov-Bohm ring of length $L$. A natural extension of these experiments 4 is to consider not only the conductance but also finite-size effects observable in the persistent current generated in the presence of a magnetic flux, $\Phi$.

The persistent current in quantum dot systems operated in the Kondo limit has been the topic of a series of recent theoretical studies $, \frac{19}{2}, 20,21,22,23,24,25,26,27,28,29,30$ Usually, two fundamentally different geometries are considered; $\stackrel{26}{\underline{w}}$ the embedded quantum $\operatorname{dot}(\mathrm{EQD})$ and the side-coupled quantum dot (SCQD) and it has been clearly established that the persistent current has a strong dependence on the parity of the total number of electrons, $N$, in the system. Here we shall exclusively be concerned with the SCQD. Suppressing electron spin indices, the Hamiltonian is in this case:

$$
H_{\mathrm{SCQD}}=-t \sum_{j=0}^{L-1}\left(c_{j}^{\dagger} c_{j+1}+\text { h.c. }\right)+H_{K}, \quad\left(c_{L} \equiv c_{0}\right)
$$

where $H_{K}=J_{K} \vec{S} \cdot c_{0}^{\dagger} \frac{\vec{\sigma}}{2} c_{0}$, and $c_{j \sigma}$ is the electron annihilation operator at site $j$ for spin $\sigma$ and $S^{a}$ are $\mathrm{S}=1 / 2$ spin operators. In the following we set $t=1$. We always include the impurity spin as an electron in our definition of $N$ but do not count the impurity site in our definition of $L$. Hence, at half-filling we have $N=L+1$. The dimensionless magnetic flux, $\alpha \equiv e \Phi / c$ is introduced by appropriately changing the phases of the hopping terms at the corresponding sites. (We set $\hbar=1$.) The persistent current is given by $j=-e d E_{0} / d \alpha$ where $E_{0}$ is the ground-state energy. Despite the simplicity of this geometry, a complete understanding of the behavior of the 
persistent current both at half-filling and off half-filling has proved exceedingly difficult to achieve. In previous work $^{25,26}$ by one of us and P. Simon it was predicted that, $j L /\left(e v_{F}\right) \rightarrow 0$ when $\xi_{K} / L \rightarrow 0$ for both parities of $N$ in contrast to the results of Refs 23,24 . However, subsequent work $^{30}$ confirmed this prediction at half-filling but showed both analytical and numerical evidence for additional terms off half-filling for $N$ even.

In the present paper we develop a Fermi liquid theory (FLT) for the persistent current past a SCQD, valid for $1 \ll \xi_{K} \ll L$. The theory provides independent confirmation of the above mentioned prediction at halffilling 25,26 as well as additional analytical evidence for the additional terms off half-filling for $N$ even. The theory yields detailed predictions about the finite-size $L$ dependence of the first two harmonics of the persistent current, using a single parameter, $1 / T_{K}$. Section [II outlines the FLT in considerable detail while section III A presents our numerical results at half-filling and section IIIB our numerical results off half-filling. In Sec. IV we calculate the finite temperature conductance past the quantum dot, at $T \ll T_{K}$. This enables us to calculate a generalized "Wilson ratio" relating the persistent current for a finite ring at $T=0$ with the conductance at finite $T$ for the same quantum dot side-coupled to infinite leads. This could be useful in an experimental context since a prior infinite leads measurement of the low $T$ conductance would fix the precise numerical coefficient in the finite size dependence of the persistent current at $T=0$.

\section{FERMI LIQUID THEORY FOR THE PERSISTENT CURRENT}

Kondo physics at low energy scales and long length scales, $T \ll T_{K}, L \gg \xi_{K}$, can be described by an effective Hamiltonian not containing the impurity spin, since it is screened by the conduction electrons and the energy scale associated with breaking this spin singlet is of $O\left(T_{K}\right)$. The low energy quasi-particles propogate freely except that they obey a modified boundary condition which can be thought of as arising because they must stay in wave-functions orthogonal to that of the screening electron. This modified boundary condition is equivalent to a $\pi / 2$ phase shift for even-channel electrons (or for s-wave electrons in the three dimensional, spherically symmetric version of the model). In addition to the modified boundary condition an interaction term is generated in this effective Hamiltonian which is only non-vanishing near the impurity location, at $|x|<\xi_{K}$. In a long wavelength effective Hamiltonian this interaction appears right at the origin. This interaction is irrelevant in the renormalization group sense; treating it in perturbation theory, its effects become weaker and weaker at lower energies and longer lengths. Building on earlier work by Wilson,,$\frac{31}{=}$ Nozières 10 developed an elegant description of this physics, following the Landau approach to Fermi liquid theory, by writing an expression for the phase shift which depends on the energy of the electron being scattered and also on the local density of electons at the origin. In 32 this approach was transcribed into an effective Hamiltonian, an approach which is simpler to deal with in some situations and more in accord with modern renormalization group (RG) techniques. (In a similar way, the Landau approach to Fermi liquids can be recast in terms of an effective Hamiltonian which can be studied by RG methods. See, for example, 33].) Furthermore, in 32 it was shown how this effective Hamiltonian could be uniquely determined, up to one overall coupling constant with dimensions of inverse energy, $1 / T_{K}$, by using the one-dimensional (1D) formulation of the Kondo model and 1D spin-charge separation. Essentially all low energy long distance properties of the Kondo model can be calculated by perturbation theory in this Fermi liquid interaction. They are generally proportional to the first or second power of the coupling constant, $1 / T_{K}$ or $1 / T_{K}^{2}$. It is not an easy matter to determined the precise value of $T_{K}$ for a given microscopic model. However, for a weak bare Kondo coupling, $\nu J \ll 1$ (where $\nu$ is the density of states) it is known that $T_{K}$ is exponentially small, $T_{K} \propto D \exp [-1 /(J \nu)]$, where $D$ is a cut-off or bandwidth scale. Furthermore, when the bare coupling is weak, the physics is universal at all energy scales $\ll D$. This implies, in particular, that the ratios of various physical quantities calculated in perturbation theory in $1 / T_{K}$ are universal. The most famous such universal ratio, between the impurity susceptibility and specific heat, is known as the Wilson ratio. However, many more such universal ratios can be readily calculated. In this section we extend extend this approach to the persistent current.

\section{A. The Boundary Conditions}

Since Fermi liquid theory applies at low energies and long distances, it is convenient to linearize the spectrum around the Fermi points $\pm k_{F}$ and to introduce left (L) and right $(\mathrm{R})$ moving chiral fields:

$$
c_{x} \sim e^{i k_{F} x} \psi_{R}(x)+e^{-i k_{F} x} \psi_{L}(x) .
$$

The non-interacting part of the continuum Hamiltonian becomes:

$$
H_{0}=\int_{0}^{L}\left[\psi_{R}^{\dagger} i \partial_{x} \psi_{R}-\psi_{L}^{\dagger} i \partial_{x} \psi_{L}\right] .
$$

We have set $v_{F}=1$. The Kondo interaction becomes:

$$
H_{K} \rightarrow J_{K} \vec{S} \cdot\left[\psi_{R}^{\dagger}(0)+\psi_{L}^{\dagger}(0)\right] \frac{\vec{\sigma}}{2}\left[\psi_{R}(0)+\psi_{L}(0)\right] .
$$

The strong coupling fixed point of the Kondo problem corresponds to the boundary condition:

$$
c_{0} \propto \psi_{L}(0)+\psi_{R}(0)=0 .
$$

We may think of the sceening electron as sitting at the origin, $j=0$ and the other electrons (low energy quasiparticles) must not enter or leave the origin in order to 
avoid breaking up the Kondo singlet. Considering the model defined on a ring, and ignoring, for the moment, the magnetic flux, there is a second boundary condition at the strong coupling fixed point:

$$
c_{L} \propto e^{i k_{F} L} \psi_{R}(L)+e^{-i k_{F} L} \psi_{R}(L)=0 .
$$

This strong coupling fixed point corresponds to an open chain of $L-1$ sites $[j=1,2,3, \ldots(L-1)]$ with no impurity spin. Initially we consider half-filling. In this case, for such an open chain, $k_{F}=\pi / 2$ regardless of the parity of $L$. Since the boundary conditions of Eqs. 2.4 2.5 are true at all times, while $\psi_{R / L}$ is a function of $t \mp x$ only, it follows from Eq. (2.4) that we can regard $\psi_{R}(x)$, for $x>0$ as the analytic continuation of $\psi_{L}(x)$ to the negative $x$ axis:

$$
\psi_{R}(x)=-\psi_{L}(-x), \quad(x>0)
$$

Likewise, Eq. (2.5) implies that we can also regard $\psi_{R}(x)$ as the analytic continuation of $\psi_{L}(x)$ :

$$
\psi_{R}(L-x)=-e^{-2 i k_{F} L} \psi_{L}(L+x) . \quad(x>0)
$$

Letting $x \rightarrow L$ in Eqs. (2.7), we see that:

$$
\psi_{L}(2 L)=e^{2 i k_{F} L} \psi_{L}(0) .
$$

At half-filling this becomes:

$$
\psi_{L}(2 L)=(-1)^{L} \psi_{L}(0)
$$

periodic for $L$ even and anti-periodic for $L$ odd. We may formulate the model in terms of left-movers only with these periodic or anti-periodic boundary conditions.

\section{B. The Fermi Liquid Interaction with Zero Flux}

We now wish to write the Fermi liquid interaction in terms of this left-moving formulation of the model. This must involve the even channel fermions (even under $x \rightarrow L-x$ ) only since only the even channel appears in the Kondo interaction. Furthermore, it only involves this channel near $x=0$. Consider for example the even channel fermions in the lattice model at a distance of 1 site from the origin:

$$
c_{1}+c_{L} \propto e^{i k_{F}} \psi_{R}(1)+e^{-i k_{F}} \psi_{L}(0)+e^{i k_{F}(L-1)} \psi_{R}(L-1)+e^{-i k_{F}(L-1)} \psi_{L}(L-1)
$$

We now use the facts that $k_{F}=\pi / 2$, the continuum fields $\psi_{L}(x)$ and $\psi_{R}(x)$ are slowly varying on the lattice scale and the boundary conditions of Eq. (2.4 2.5) to write this in the purely left-moving formulation as:

$$
c_{1}+c_{L} \propto e^{-i \pi / 2} \psi_{L}(0)+e^{-i \pi(L-1) / 2} \psi_{L}(L) .
$$

We expect only this combination of fields to appear in the Fermi liquid interaction, up to higher dimension operators involving derivatives of the fermion fields. This follows from observing, using the boundary conditions of Eq. (2.4, 2.5) and the fact that the continuum fields vary slowly, that any non-vanishing even lattice fermion field near the origin is proportional to this one. For $j$ even and $j \ll \xi_{K}$ one finds:

$$
c_{j}+c_{L-j} \approx 0
$$

While for $j$ odd and $j \ll \xi_{K}$ we have:

$$
\begin{aligned}
c_{j} & +c_{L-j} \propto e^{-i \pi j / 2} \psi_{L}(0)+e^{-i \pi(L-j) / 2} \psi_{L}(L) \\
& =(-1)^{(j-1) / 2}\left[e^{-i \pi / 2} \psi_{L}(0)+e^{-i \pi(L-1) / 2} \psi_{L}(L)\right],
\end{aligned}
$$

which is proportional to Eq. (2.11).
Once we have written the Kondo interaction in terms of the even sector fermions only, it is convenient to bosonize, introducing separate spin and charge bosons. Importantly, the Kondo interaction involves only the spin bosons in the even sector. It then follows that the Fermi liquid interactions can be written in terms of these bosons only. The only possible dimension 2 operator, in the spin sector, which respects the $\mathrm{SU}(2)$ symmetry is $\vec{J}_{e}^{2}(0)$, the square of the spin density operator. Going back to the fermion representation:

$$
\vec{J}_{e}(0) \equiv \psi_{e}^{\dagger}(0) \frac{\vec{\sigma}}{2} \psi_{e}(0)
$$

where, from Eq. (2.11),

$$
\psi_{e}(0) \propto \frac{\psi_{L}(0)-e^{-i \pi L / 2} \psi_{L}(L)}{\sqrt{2}} .
$$


Thus we write the leading irrelevant Fermi liquid interaction, for the case, $L$ even as:

$$
\begin{aligned}
H_{i n t} & =\frac{-8 \pi}{3 T_{K}} \vec{J}_{e}^{2}(0) \\
& =\frac{-2 \pi}{3 T_{K}}\left\{\left[\psi_{L}(0)-e^{-i \pi L / 2} \psi_{L}(L)\right]^{\dagger} \frac{\vec{\sigma}}{2}\right. \\
& \left.\times\left[\psi_{L}(0)-e^{-i \pi L / 2} \psi_{L}(L)\right]\right\}^{2} .
\end{aligned}
$$

We have written the coupling constant in front of this operator as $8 \pi / 3 T_{K}$. It follows from standard scaling arguments that this coupling constant should be of order the RG cross-over scale. In general, it should be written as $1 / T_{K}$ times a dimensionless constant of $\mathrm{O}(1)$. All low energy propertites of the system can be determined from this interaction term, including the impurity susceptibility and specific heat, for example. The sign in Eq. (2.16) is known to be the correct one since it gives the correct (positive) sign for these two quantities. The factor of $8 \pi / 3$ is purely a matter of a convention, or a precise definition of what is meant by $T_{K}$. Unfortunately, a great number of different conventions for $T_{K}$ are in current use. We have chosen here to use the same convention as used by Nozières ${ }^{10}$ (who also referred to $1 / T_{K}$ as $\alpha$ ) and by Glazman and Pustilnik ${ }^{34}$. In Appendix B we briefly review other definitions of $T_{K}$ in popular use and the constant factors relating them to each other.

Strictly speaking, one other operator of dimension 2 is permitted, $J_{e}(0)^{2}$, the square of the charge current, rather than the spin current. As mentioned above, naively the charge sector decouples from the Kondo interactions so that this operator should apparently not be generated. However, irrelevant operators (at the weak coupling fixed point) couple charge and spin sectors together ultimately permitting this operator to occur. However, the coefficient of this operator is expected to be $O(1 / D)$, where $D$ is the bandwidth ( $t$ in the tightbinding model), rather than $O\left(1 / T_{K}\right)$. In the weak coupling limit, where $T_{K} \ll D$, this other interaction can be ignored.

\section{The Fermi Liquid Interaction with Finite Flux}

So far we haven't mentioned the flux, $\Phi=c \alpha / e$. The phases in the hopping terms corresponding to any desired flux can be inserted anywhere in the ring. They can be moved around freely by phase redefinitions of the electron fields (gauge transformations). For purposes of understanding the strong coupling fixed point it is convenient to imagine that they are inserted far from the impurity compared to $\xi_{K}$ so that the strong coupling boundary conditions, discussed above, are unaffected by the flux. Of course, this is only possible for $L \gg \xi_{K}$ but it is precisely that limit which we are now considering. The Fermi liquid theory only applies in that limit. It can be seen that adding phases to hopping terms neccessarily couples even and odd sectors together, since it breaks parity. For this reason, it is more convenient to go back to left and right movers and then ultimately to left movers only on a ring of length $2 L$, as discussed above. It is also most convenient to put the phase at the origin after establishing the strong coupling b.c. This amounts to:

$$
\psi_{L}(L) \rightarrow e^{-i \alpha} \psi_{L}(L)
$$

Hence the Ferm liquid interaction becomes:

$$
\begin{aligned}
H_{\text {int }} & =\frac{-2 \pi}{3 T_{K}}\left\{\left[\psi_{L}(0)-e^{-i(\pi L / 2+\alpha)} \psi_{L}(L)\right]^{\dagger} \frac{\vec{\sigma}}{2}\right. \\
& \left.\times\left[\psi_{L}(0)-e^{-i(\pi L / 2+\alpha)} \psi_{L}(L)\right]\right\}^{2} .
\end{aligned}
$$

\section{Perturbation Theory}

The remaining calculations are quite straightforward. We simply do first order perturbation theory in $1 / T_{K}$ for the groundstate energy, imposing periodic or antiperiodic b.c.'s, Eq. (2.9), on the purely left-moving fermion fields.

Now we turn to calculating the expectation value of $H_{\text {int }}$. From Eq. (2.18), recalling that $L=N-1$ at halffilling, it is clear that it is advantageous to define a shifted flux $\tilde{\alpha}$ in the following manner:

$$
\begin{aligned}
& \tilde{\alpha}=\alpha+\pi(N-1) / 2 \quad(\mathrm{~N} \text { odd }) \\
& \tilde{\alpha}=\alpha+\pi N / 2 \quad(\mathrm{~N} \text { even }) .
\end{aligned}
$$

[This definition can been seen largely as a way of defining the coefficient in front of $\sin (\tilde{\alpha})$ in the persistent current to be positive.] Using this definition, we first rewrite $H_{\text {int }}$ as:

$$
\begin{aligned}
& \left.H_{\text {int }}=\frac{\pi}{6 T_{K}}\left\{e^{2 i \tilde{\alpha}}\left[\psi^{\dagger}(L) \vec{\sigma} \psi(0)\right]^{2}-2 i e^{i \tilde{\alpha}} \psi^{\dagger}(L) \vec{\sigma} \psi(0) \cdot\left[\psi^{\dagger}(0) \vec{\sigma} \psi(0)+\psi^{\dagger}(L) \vec{\sigma} \psi(L)\right]+\text { h.c. }+\ldots\right\}, \quad \text { (N even }\right) \\
& H_{\text {int }}=\frac{\pi}{6 T_{K}}\left\{-e^{2 i \tilde{\alpha}}\left[\psi^{\dagger}(L) \vec{\sigma} \psi(0)\right]^{2}+2 e^{i \tilde{\alpha}} \psi^{\dagger}(L) \vec{\sigma} \psi(0) \cdot\left[\psi^{\dagger}(0) \vec{\sigma} \psi(0)+\psi^{\dagger}(L) \vec{\sigma} \psi(L)\right]+\text { h.c. }+\ldots\right\} . \quad \text { (N odd) }
\end{aligned}
$$


tribute to the current in first order in $1 / T_{K}$. The first term can be rewritten:

$$
H_{i n t}^{(1)}=\frac{\pi(-1)^{N}}{T_{K}} e^{2 i \tilde{\alpha}} \psi^{\uparrow \dagger}(L) \psi_{\uparrow}(0) \psi^{\downarrow \dagger}(L) \psi_{\downarrow}(0)+\text { h.c. }
$$

We now evaluate the various terms using Wick's theorem. For this we need the equal time propogator for a left mover with anti-periodic and periodic boundary conditions on an interval of length $2 L$.

\section{1. $\quad N$ Even at Half-filling}

For the case $N$ even, with anti-periodic boundary conditions, this is:

$$
\begin{aligned}
<\psi^{\dagger \alpha}(x) \psi_{\beta}(0)> & =\delta_{\beta}^{\alpha} \frac{1}{2 L} \sum_{0}^{\infty} e^{-i \pi(m+1 / 2) x / L} \\
& =\frac{-i \delta_{\beta}^{\alpha}}{4 L \sin (\pi x / 2 L)}
\end{aligned}
$$

In particular:

$$
<\psi^{\dagger \alpha}(L) \psi_{\beta}(0)>=\frac{-i \delta_{\beta}^{\alpha}}{4 L}
$$

Of course,

$$
<\psi^{\dagger \alpha}(L) \psi_{\beta}(0)>=-<\psi_{\beta}(0) \psi^{\dagger \alpha}(L)>.
$$

We also need an expression for $<\psi^{\dagger \alpha}(0) \psi_{\beta}(0)>$. The sum in Eq. (2.22) is ultraviolet divergent so we introduce a (dimensionless) cut-off, an upper bound on the summation variable $m: m<D$, giving:

$$
<\psi^{\dagger \alpha}(0) \psi_{\beta}(0)>=\frac{\delta_{\beta}^{\alpha} D}{2 L} .
$$

We will see that our results for the current do not depend on the value of $D$. It follows from $\mathrm{PH}$ symmetry that:

$$
<\psi_{\beta}(0) \psi^{\dagger \alpha}(0)>=\frac{\delta_{\beta}^{\alpha} D}{2 L}
$$

Furthermore

$$
<\psi_{\beta}(0) \psi^{\dagger \alpha}(0)>=<\psi_{\beta}(L) \psi^{\dagger \alpha}(L)>
$$

as follows from the reflection symmetry of the model which takes $x \rightarrow L-x$.

The $\cos 2 \alpha$ term is:

$$
E_{0}^{(2)}=\frac{-\pi}{T_{K}} e^{2 i \tilde{\alpha}}\left(\frac{-i}{4 L}\right)^{2}+h . c .=\frac{\pi}{8 T_{K} L^{2}} \cos 2 \tilde{\alpha},
$$

With a current:

$$
j^{(2)}=-e d E_{0} / d \tilde{\alpha}=\frac{e \pi}{4 T_{K} L^{2}} \sin 2 \tilde{\alpha}(N \text { even }) .
$$

Now consider the $\cos \tilde{\alpha}$ term in the energy. This is actually zero for $N$ even but let's go through some steps which are useful also for $N$ odd. We use Wick's theorem to write this as:

$$
\begin{aligned}
& <\psi^{\dagger}(L) \vec{\sigma} \psi(0) \cdot\left[\psi^{\dagger}(0) \vec{\sigma} \psi(0)+\psi^{\dagger}(L) \vec{\sigma} \psi(L)\right]> \\
& =2<\psi^{\dagger}(L) \vec{\sigma} \psi(0)>\cdot<\psi^{\dagger}(0) \vec{\sigma} \psi(0)> \\
& +<\psi^{\alpha \dagger}(L) \psi_{\delta}(0)><\psi_{\beta}(0) \psi^{\gamma \dagger}(0)>\vec{\sigma}_{\alpha}^{\beta} \cdot \vec{\sigma}_{\gamma}^{\delta} \\
& +<\psi^{\alpha \dagger}(L) \psi_{\delta}(L)><\psi_{\beta}(0) \psi^{\gamma \dagger}(L)>\vec{\sigma}_{\alpha}^{\beta} \cdot \vec{\sigma}_{\gamma}^{\delta}
\end{aligned}
$$

For $N$ even it is easy to see that the first term in Eq. (2.30) vanishes and the last two cancel using Eqs. (2.23)(2.27).

\section{2. $\quad$ Odd at Half-filling}

Now consider $N$ odd. The strong coupling groundstate has spin $-1 / 2$; we choose the state with total $S^{z}=1 / 2$. The propogators are now different for spin up or down. We find:

$$
<\psi^{\uparrow \dagger}(x) \psi_{\uparrow}(0)>=\frac{1}{2 L} \sum_{m=0}^{\infty} e^{-i \pi m x / L}=\frac{-i}{4 L} \frac{e^{i \pi x / 2 L}}{\sin (\pi x / 2 L)} .
$$

On the other hand, the $\downarrow$ propagator is the same except that the $m=0$ term is omitted from the sum, subtracting $1 / 2 L$ to the final result. Thus:

$$
<\psi^{\alpha \dagger}(L) \psi_{\beta}(0)>=\frac{\left(\sigma^{z}\right)_{\beta}^{\alpha}}{4 L} .
$$

Again we impose a cut-off so:

$$
<\psi^{\uparrow \dagger}(0) \psi_{\uparrow}(0)>=\frac{D}{2 L} .
$$

Now we have:

$$
<\psi_{\uparrow}(0) \psi^{\uparrow \dagger}(0)>=\frac{D-1}{2 L} .
$$

The $(-1)$ reflects the breaking of particle-hole (P-H) symmetry. We have one unpaired spin up electron, sitting right at the Fermi surface. Furthermore,

$$
<\psi^{\downarrow \dagger}(0) \psi_{\downarrow}(0)>=\frac{D-1}{2 L}
$$

and

$$
<\psi_{\downarrow}(0) \psi^{\downarrow \dagger}(0)>=\frac{D}{2 L} .
$$

We may combine these as:

$$
\begin{aligned}
& <\psi^{\alpha \dagger}(0) \psi_{\beta}(0)>=\frac{D-1 / 2}{2 L} \delta_{\beta}^{\alpha}+\frac{1}{4 L}\left(\sigma^{z}\right)_{\beta}^{\alpha} \\
& <\psi_{\alpha}(0) \psi^{\beta \dagger}(0)>=\frac{D-1 / 2}{2 L} \delta_{\alpha}^{\beta}-\frac{1}{4 L}\left(\sigma^{z}\right)_{\beta}^{\alpha}
\end{aligned}
$$


We now substitute this into Eq. (2.30). It is easy to see that the terms containing $D$ all cancel, for the same reasons that the entire expression vanishes for $N$ even. The remaining terms are:

$$
\begin{aligned}
& <\psi^{\dagger}(L) \vec{\sigma} \psi(0) \cdot\left[\psi^{\dagger}(0) \vec{\sigma} \psi(0)+\psi^{\dagger}(L) \vec{\sigma} \psi(L)\right]> \\
& =\left(\frac{1}{4 L}\right)^{2}\left[2 \operatorname{tr}\left(\vec{\sigma} \sigma^{z}\right) \cdot \operatorname{tr}\left(\vec{\sigma} \sigma^{z}\right)-2 \operatorname{tr}\left(\vec{\sigma} \sigma^{z} \cdot \vec{\sigma} \sigma^{z}\right)\right] \\
& =\left(\frac{1}{4 L}\right)^{2}[8+4] \\
& =\frac{3}{4 L^{2}}
\end{aligned}
$$

Thus the $\cos \tilde{\alpha}$ term in the energy, for $N$ odd, is:

$$
E_{0}^{(1)}=\frac{\pi}{6 T_{K}} 2 e^{i \tilde{\alpha}} \frac{3}{4 L^{2}}+\text { c.c. }=\frac{\pi}{2 T_{K} L^{2}} \cos \tilde{\alpha} .
$$

The term in the current is:

$$
j^{(1)}=\frac{e \pi}{2 T_{K} L^{2}} \sin \tilde{\alpha}(N \text { odd }) .
$$

The $\sin (2 \tilde{\alpha})$ in the current for odd $N$ is given again by Eq. (2.29), with the opposite sign:

$$
j^{(2)}=-\frac{e \pi}{4 T_{K} L^{2}} \sin 2 \tilde{\alpha}(N \text { odd }) .
$$

Note that the minus sign which follows from spin up and down Green's functions having opposite sign for $N$ odd, Eq. (2.32), replaces the minus sign due to the factors of $i$ in the Green's function for $N$ even, Eq. (2.23), leaving only the minus sign from Eq. (2.20).

We have so far set $v_{F}=1$. We summarize our results on the persistent current, for $\xi_{K} \ll L$ below, reinserting a factor of $v_{F}^{2}$ by dimensional analysis and replacing $v_{F} / T_{K}$ by $\xi_{K}$ :

$$
\begin{aligned}
& j_{e} \rightarrow \frac{e v_{F}}{L} \frac{\xi_{K}}{L} \frac{\pi}{4} \sin 2 \tilde{\alpha} \\
& j_{o} \rightarrow \frac{e v_{F}}{L} \frac{\xi_{K}}{L}\left[\frac{\pi}{2} \sin \tilde{\alpha}-\frac{\pi}{4} \sin 2 \tilde{\alpha}\right] .
\end{aligned}
$$

It was argued earlier 25 , that both $j_{e}$ and $j_{o}$ can be written as $e v_{F} / L$ times universal scaling functions of $\xi_{K} / L$ and $\tilde{\alpha}$. Eq. (2.42) indeed has that form.

\section{Off Half-filling}

Now consider the case away from $1 / 2$-filling, where P-H symmetry is broken. Here we refer to particle-hole symmetry breaking by an amount of $O(1)$ not an amount of $O(1 / L)$ as occurs, even at $1 / 2$-filling for $N$ even. Note that we continue to choose the reduced band to be symmetric around $k_{F}: k_{F}-\Lambda \leq k \leq k_{F}+\Lambda$. However, the process of integrating out wave-vectors further away from $k_{F}$ is not $\mathrm{P}-\mathrm{H}$ symmetric, when $k_{F}$ does not have the $\mathrm{P}-\mathrm{H}$ symmetric value, so we expect to generate $\mathrm{PH}$ symmetry breaking terms in $H_{\text {int }}$. The most important such term, which can lead to a current of $O(1 / L)$, is:

$$
H_{2}=-e^{i \pi(N-1) / 2} \lambda e^{i \alpha} \psi^{\dagger}(L) \psi(0)+\text { h.c. }
$$

where $\lambda$ is a real coupling constant. Here the phase in this expression is determined by parity, i.e. by the fact that it arises from a term $-2 \lambda \psi_{e}^{\dagger} \psi_{e}$ using Eq. (2.11). This term only occurs when P-H symmetry is broken. We expect $\lambda \propto\left(J_{K} / t\right)^{2}$ where $t$ is the original bandwidth.

More generally, we might start with a microscopic model which includes second neighbor tunnelling past the quantum dot:

$$
\delta H=-t_{2}\left(c_{L-1}^{\dagger} c_{1}+\text { h.c. }\right) .
$$

Such a term breaks P-H symmetry even at half-filling and leads to a term in the low energy effective Hamiltonian of the form of $\mathrm{H}_{2}$ in Eq. (2.43) with $\lambda \propto t_{2}$.

If the system is very close to half-filling, $n-1=\delta n \ll$ $1 / \xi_{K}$, and we begin with a pure Kondo model with no potential scattering or direct tunnelling, then we can calculate the value of $\lambda$, in $H_{2}$ directly from the Fermi liquid interaction $H_{\text {int }}$ of Eq. (2.20). To do this, it is convenient to integrate out wave-vectors in the RG transformation symmetrically around $k=\pi / 2$ even thought this is no longer $k_{F}$, which has the value:

$$
k_{F}=(\pi / 2)(1+\delta n)
$$

In this way, $\lambda$ is not generated during the $\mathrm{RG}$ transformation, and we obtain only the interaction $H_{\text {int }}$. However, the low energy effective theory inherits an asymmetric cut-off:

$$
k_{F}-\Lambda-(\pi / 2) \delta n<k<k_{F}-(\pi / 2) \delta n+\Lambda .
$$

We may now generate $H_{2}$ from $H_{\text {int }}$ by simply normal ordering $H_{\text {int }}$. This follows since Eqs. (2.25, 2.26) are now modified to:

$$
\begin{aligned}
& <\psi^{\dagger \alpha}(0) \psi_{\beta}(0)>=\delta_{\beta}^{\alpha}\left[\frac{D}{2 L}+\frac{\delta n}{4}\right] \\
& <\psi_{\beta}(0) \psi^{\dagger \alpha}(0)>=\delta_{\beta}^{\alpha}\left[\frac{D}{2 L}-\frac{\delta n}{4}\right]
\end{aligned}
$$

We now write $H_{\text {int }}$ as a normal ordered part plus a correction of the form of $\mathrm{H}_{2}$. This follows using: 


$$
\begin{aligned}
& \psi^{\dagger}(L) \vec{\sigma} \psi(0) \cdot\left[\psi^{\dagger}(0) \vec{\sigma} \psi(0)+\psi^{\dagger}(L) \vec{\sigma} \psi(L)\right]=: \psi^{\dagger}(L) \vec{\sigma} \psi(0) \cdot\left[\psi^{\dagger}(0) \vec{\sigma} \psi(0)+\psi^{\dagger}(L) \vec{\sigma} \psi(L)\right]: \\
+ & {\left[\psi^{\alpha \dagger}(L) \psi_{\delta}(0)<0\left|\psi_{\beta}(0) \psi^{\gamma \dagger}(0)\right| 0>-\psi^{\gamma \dagger}(L) \psi_{\beta}(0)<0 \mid \psi^{\alpha \dagger}(L) \psi_{\delta}(L)\right]\left(\vec{\sigma}_{\alpha}^{\beta} \cdot \vec{\sigma}_{\gamma}^{\delta}\right) . }
\end{aligned}
$$

Using Eq. (2.47) we see that the two terms in the second line of Eq. (2.48) don't cancel, away from half-filling, yielding instead:

$$
\psi^{\dagger}(L) \vec{\sigma} \psi(0) \cdot\left[\psi^{\dagger}(0) \vec{\sigma} \psi(0)+\psi^{\dagger}(L) \vec{\sigma} \psi(L)\right]=: \psi^{\dagger}(L) \vec{\sigma} \psi(0) \cdot\left[\psi^{\dagger}(0) \vec{\sigma} \psi(0)+\psi^{\dagger}(L) \vec{\sigma} \psi(L)\right]:-\frac{3 \delta n}{2} \psi^{\dagger}(L) \psi(0)
$$

Inserting this expression into Eq. (2.20) gives a quadratic term of the form of $\mathrm{H}_{2}$ with:

$$
\lambda=\frac{\pi \delta n}{2 T_{K}}
$$

for $N$ even.

We may now evaluate the additional terms in the current arising from $\mathrm{H}_{2}$, using perturbation theory in $H_{\text {int }}$. We only discuss first order perturbation theory. We evaluate $<H_{2}>$ using Eq. (2.23). This gives an additional current:

$$
\delta j=(e \lambda / L) \sin \tilde{\alpha},
$$

for $N$ even. For $N$ odd, there is no extra contribution to first order in $\lambda$ since $<\psi^{\dagger \alpha}(L) \psi_{\beta}(0)>\propto\left(\sigma^{z}\right)_{\beta}^{\alpha}$. Hence, to first order, we expect that in the limit of small $\delta n$ the current for $N$ odd, $j_{o}$, remain unchanged with respect to it's value at half-filling.

Note however, that we have only evaluated the contributions of $H_{\text {int }}$ to the current in first order perturbation theory in $\mathrm{H}_{2}$. Thus we haven't ruled out the possibility of a term of $O(1 / L)$ in the current for $N$ odd, with broken PH symmetry.

Adding the universal result for the $\mathrm{PH}$ symmetric case to this correction from PH symmetry breaking (and reinserting the factor of $v_{F}$ previously set to one) gives a persistent current, for $N$ even:

$$
j_{e}=\frac{e v_{F}}{L}\left[\frac{\pi \xi_{K}}{4 L} \sin (2 \tilde{\alpha})+\lambda \sin (\tilde{\alpha})\right] .
$$

The non-universal term arising from $\mathrm{PH}$ symmetry breaking, proportional to $\lambda$ will always dominate at sufficiently large $L$. However, provided that the dimensionless coupling, $\lambda \ll 1$, as we expect for small bare Kondo coupling and not too strong direct tunnelling across the quantum dot, the first, universal, term will dominate over the range of lengths:

$$
\xi_{K} \ll L \ll \xi_{K} / \lambda .
$$

The presence of a term in $j_{e}$ proportional to $\sin (\tilde{\alpha})$ in the absence of PH symmetry was shown in Ref. 30 using strong coupling perturbation theory. The above derivation provides further independent analytical evidence for such a term.

\section{NUMERICAL RESULTS}

The Fermi liquid theory results for the persistent current developed in the previous section are parameterized in terms of $T_{K}$ and should be valid in the regime $T \ll T_{K}$, $1 \ll \xi_{K} \ll L$. For a useful numerical test of these results it is therefore necessary to study ratios of the Fourier components of the persistent current that become independent of $T_{K}$ and can be tested at $T=0$ using exact diagonalization (ED) methods. Furthermore, in order for $1 \ll \xi_{K}$ to hold, $J_{K}$ should not be too large. A more severe constraint is that the system sizes should satisfy $L \gg \xi_{K}$. With an exponentially diverging $\xi_{K}$ at small $J_{K}$ we see that we quickly leave the regime of validity of Fermi liquid theory as $J_{K} \rightarrow 0$ for the rather modest systems sizes we can treat using ED. Clearly, the interesting regime is then intermediate values for $J_{K}$. Our approach is then to calculate the different Fourier components, $a_{n}$, of the persistent current, $j$, as a function of the flux, $\alpha$. Our convention for the Fourier components are $j(\tilde{\alpha})=\sum_{n} a_{n} \sin (n \tilde{\alpha})$. Note that, a very precise determination of the $\alpha$ dependence is necessary in order to determine the Fourier components reliably and we typically use 200 values for $\alpha$. The numerical results can then be compared to the predictions of the Fermi liquid theory as well as results previously obtained using strong coupling perturbation $\underline{30}^{30}$

\section{A. At Half-filling}

We start by discussing our numerical ED results obtained at half-filling with systems sizes of $L=12-15$. 


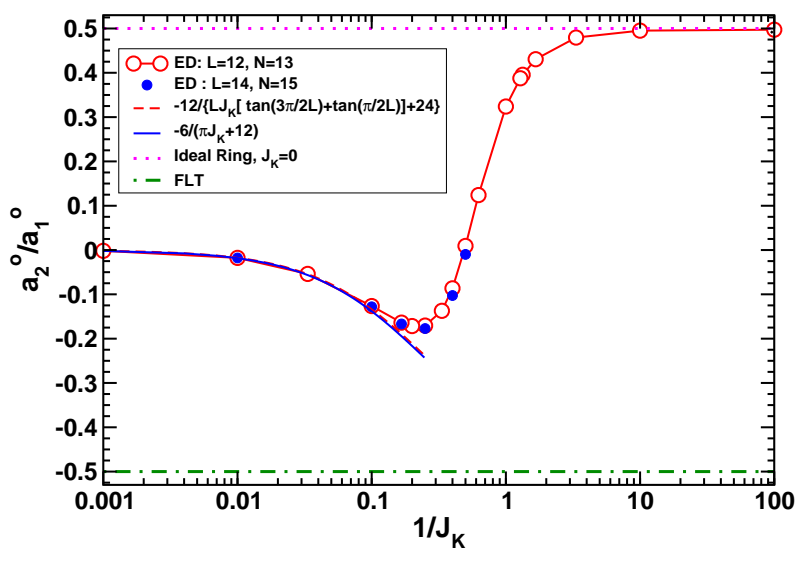

FIG. 1: The ratio, $a_{2}^{o} / a_{1}^{o}$, of the two first Fourier coefficients of the current for the SCQD at 1/2-filling, for $N$ odd. $\circ$ indicates ED results with $L=12$ and $N=13$ while $\bullet$ indicates ED results with $L=14$ and $N=15$. The dashed line (barely visible) is the strong coupling perturbative result, Eq. (3.2), for this ratio with $L=12$, the solid line is $L \rightarrow \infty$ the strong coupling perturbative result, Eq. (3.3), the dashed dotted line the fermi liquid result, Eq. (3.4), and the dotted line the ideal ring result, Eq. (3.5).

Due to the lack of symmetry in these systems it is difficult to reach larger sizes and the largest system $(L=$ $14, N=15)$ required a diagonalization of a matrix of size $20,796,633$ for each value of $\alpha$ after symmetry reductions.

In Ref 30 strong coupling perturbative results were given for the persistent current at half-filling past the SCQD. It was shown that, for odd or even $N$,

$$
\begin{aligned}
j_{o} L / e \approx & \frac{32}{9 J_{K}^{2}}\left[\tan \left(\frac{\pi}{2 L}\right)+\tan \left(\frac{3 \pi}{2 L}\right)\right] \sin \tilde{\alpha} \\
& +\frac{128}{3 J_{K}^{3} L}[2 \sin \tilde{\alpha}-\sin (2 \tilde{\alpha})] \\
j_{e} L / e \approx & \frac{32}{3 J_{K}^{3} L}[1+1 / \cos (\pi / L)]^{2} \sin 2 \tilde{\alpha} .
\end{aligned}
$$

As elsewhere, $L$ does not include the impurity site while $N$ does include the impurity electron. The definition of $\tilde{\alpha}$ is also the same: For $N$ odd $\tilde{\alpha}=\alpha+\pi(N-1) / 2$ and for $N$ even $\tilde{\alpha}=\alpha+\pi N / 2$. If we concentrate on the first two Fourier coefficients of the current we see from the above strong coupling results of Eq. (3.1) that:

$$
\begin{aligned}
& \frac{a_{2}^{o}}{a_{1}^{o}}=\frac{-12}{J_{K} L[\tan (\pi / 2 L)+\tan (3 \pi / 2 L)]+24} \\
& \frac{a_{2}^{o}}{a_{2}^{e}}=\frac{-4}{\left(1+1 / \cos \left(\pi / L^{e}\right)\right)^{2}} \frac{L^{e}}{L^{o}} .
\end{aligned}
$$

Here the superscripts o and e refer to $N$ odd or even, respectively. In the limit $L \rightarrow \infty$ we see that the strong coupling expansion gives:

$$
\frac{a_{2}^{o}}{a_{1}^{o}} \rightarrow \frac{-6}{\pi J_{K}+12}, \quad \frac{a_{2}^{o}}{a_{2}^{e}} \rightarrow-1 .
$$

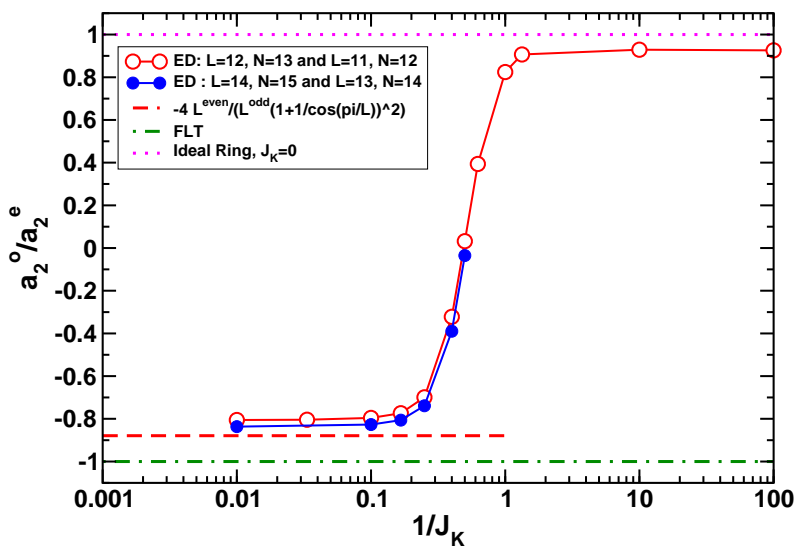

FIG. 2: The ratio, $a_{2}^{o} / a_{2}^{e}$, of the second Fourier coefficients of the current for the SCQD at 1/2-filling, for $N$ odd and $N$ even. $\circ$ indicates ED results with $L=11, N=12$ and $L=12, N=13$ while $\bullet$ indicates ED results with $L=13$, $N=14$ and $L=14, N=15$. The dashed line is the strong coupling perturbative result, Eq.(3.2), for this ratio with $L^{\text {even }}=11, L^{\text {odd }}=12$, the dashed dotted line the fermi liquid result, Eq. (3.4), and the dotted line the ideal ring result, Eq. (3.5).

The Fermi liquid theory developed in the preceeding section, valid for $1 \ll \xi_{K} \ll L$, predicts, using Eqs. (2.29) and (2.40):

$$
\frac{a_{2}^{o}}{a_{1}^{o}}=-\frac{1}{2}, \quad \frac{a_{2}^{o}}{a_{2}^{e}}=-1
$$

When $J_{K}$ is exactly zero we obtain the behavior of an ideal ring, in this case the current has a charateristic sawtooth like form 25,35 where the same Fourier coefficients can be trivially calculated. One finds at $J_{K}=0$ :

$$
\frac{a_{2}^{o}}{a_{1}^{o}}=\frac{1}{2}, \quad \frac{a_{2}^{o}}{a_{2}^{e}}=1 .
$$

Note that this result is dramatically different from the result of Eq. (3.4), valid for $1 \ll \xi_{K} \ll L$. The ratio of the Fourier coefficients appear discontinuous in the limit $J_{K} \rightarrow 0$.

We begin by discussing our results for $a_{2}^{o} / a_{1}^{o}$ which are shown in Fig. 1 for system sizes of $L=12$ and $L=14$, for a range of different $J_{K}$. The theoretical strong coupling result of Eq. (3.2) for $L=12$ is shown as the dashed line and differs only slightly from the $L \rightarrow \infty$ result of Eq. (3.3) shown as the solid line. The numerical results agrees with the strong coupling results once $J_{K}>10$. In the opposite limit, $J_{K} \rightarrow 0$, we see that the numerical results quickly approach the ideal ring result of Eq. (3.5), shown as the dotted line. This is very reasonable, since the very limited system sizes of $L=12,14$ certainly no longer satisfies the equality $L \gg \xi_{K}$ for Fermi liquid theory to be valid once $\xi_{K}$ diverges as $J_{K} \rightarrow 0$. The interesting region is therefore the intermediate coupling 
region, $0.1<J_{K}<10$. A very rapid crossover from the ideal ring result of $1 / 2$, at small $J_{K}$, to a negative value is observed, consistent with an exponentially diverging $\xi_{K}$. Furthermore, $a_{2}^{o} / a_{1}^{o}$ develops a pronounced plateau ("dip") around $J_{K} \sim 4$, reaching negative values, even for these rather modest system sizes. As we increase the system size from $L=12$ to $L=14$ this "dip" becomes slightly more pronounced. This implies that the crossover between the ideal ring result for this ratio of $1 / 2$, at small $J_{K}$, to the $J_{K} \rightarrow \infty$ result of 0 , can not be monotonic even in the thermodynamic limit. We take this to be indicative of the validity of the Fermi Liquid theory. We expect that numerical results for $a_{2}^{o} / a_{1}^{o}$ in the thermodynamic limit would roughly follow the strong coupling result given by Eq. (3.3) (the solid line in Fig. 1) out to $J_{K} \sim 0.1$ and then attain the value $-1 / 2$ rather quickly, jumping discontinuously for $J_{K}=0$, in accordance with the Fermi Liquid theory. In the subsequent section, where we discuss our results away from half-filling, we therefore exclusively focus on the value of $J_{K}=4$ where the "dip" occurs, since this would appear to be the most promising value for $J_{K}$ for observing FLT behavior with the available system sizes.

Results for $a_{2}^{o} / a_{2}^{e}$ are shown in Fig. 2. In this case the behavior as a function of $J_{K}$ is monotonic and the evidence for a regime described by FLT perhaps less obvious. The dotted, dashed and dashed-dotted lines are the ideal ring results of Eq. (3.5), the strong coupling result of Eq. (3.2) and the FLT result of Eq. (3.4). Again an extremely rapid crossover is seen at intermediate values of $J_{K}$. Furthermore, as the system size is increased from $L=12$ to $L=14$ this crossover moves to smaller values of $J_{K}$ approaching the predictions of Fermi Liquid theory.

\section{B. Off Half-filling}

Next, we turn to a discussion of our results for off halffilling. With our definitions of $N$ and $L$ we define, as elsewhere, $\delta n=(N-1) / L-1$. A typical Hilbert space size is $64,414,350$ for ( $L=15, N=14$ ) after symmetry reductions. In Ref 30 strong coupling perturbative results for the current past the SCQD away from half-filling were also given. At large $J_{K}$ it was found that for $N$ odd and $N$ even:

$$
\begin{aligned}
& j_{o} L / e \approx \frac{32}{9 J_{K}^{2}} \sin (\tilde{\alpha}) \\
& \times\left[\sin \frac{\pi(N-1)}{2 L} \tan \frac{\pi}{2 L}-\sin \frac{3 \pi(N-1)}{2 L} \tan \frac{3 \pi}{2 L}\right] \\
& j_{e} L / e \approx \frac{32}{9 J_{K}^{2}} \sin (\tilde{\alpha}) \\
& \times\left[\frac{\cos (\pi(N-1) /(2 L))}{\cos (\pi /(2 L))}-\frac{\cos (3 \pi(N-1) /(2 L))}{\cos (3 \pi /(2 L))}\right]
\end{aligned}
$$

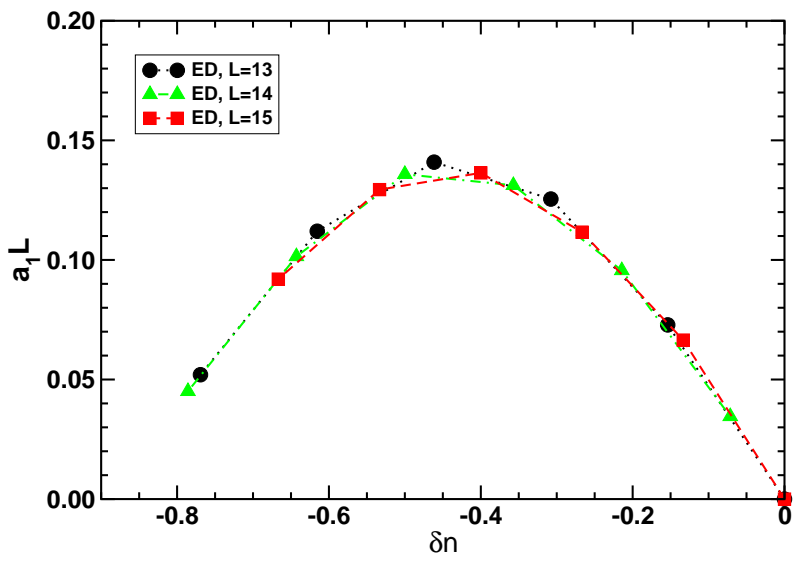

FIG. 3: The first Fourier coefficient scaled by $L, a_{1}^{e} L$, of the current for the SCQD away from 1/2-filling, for $N$ even as a function of $\delta n=(N-1) / L-1$. In all cases $J_{K}=4.0$. $\circ$ indicates ED results with $L=13$ and $N=14,12,10,8,6,4, \triangle$ indicates ED results with $L=14$ and $N=14,12,10,8,6,4$, finally - indicates ED results with $L=15$ and $N=$ $16,14,12,10,8,6$. The lines are guides to the eye.

This results predicts that as $L \rightarrow \infty, \delta n \rightarrow 0, j_{o} L / e$ is changed from the value at half-filling, Eq. (3.1), only by a term proportional to $-(\delta n)^{2} \sin (\tilde{\alpha}) /\left(J_{K}^{2} L\right)$. If we only work to linear order in $\delta n$ this correction can be neglected. However in this limit, $j_{e} L / e \sim$ $\sin (\tilde{\alpha}) 64 \pi \delta n / 9 J_{K}^{2}$, that is, a $\sin (\tilde{\alpha})$ term proportional to $\delta n$. At half-filling this term is clearly 0 for $j_{e}$, and the $\sin (2 \tilde{\alpha})$ term in Eq. (3.1) dominates. We note that this limit of the strong coupling results agrees with the previously developed Fermi liquid theory that, in the limit $\delta n \rightarrow 0$, predicted no change in $j_{o}$ but the generation of a $\sin (\tilde{\alpha})$ term proportional to $\delta n / L$ for $j_{e}$, Eq. (2.50), (2.51). In the following, we therefore focus exclusively on the first Fourier coefficient, $a_{1}$, determining its dependence on $\delta n$.

Our results for $a_{1}^{e} L$ for $N$ even are shown in Fig. 3 for system sizes $L=13,14,15$ for a range of $\delta n$. All results are for an intermediate coupling of $J_{K}=4$, the most promising coupling to show clear indications of FLT behavior for our limited system sizes. Two conclusions are immediately evident; since the results fall on a single curve this term in the current is indeed proportional to $1 / L$ as predicted by theory. Secondly, for small $\delta n$, $a_{1} L$ increases approximately linearly with $\delta n$, again consistent with the theoretical predictions. At larger $\delta n$ the results strongly deviate from linear behavior as one would expect.

Finally, we show results for $a_{1}^{o} L^{2}$ for $N$ odd in Fig. 4 for system sizes of $L=12,13,14$, for a range of $\delta n$. The results are again for an intermediate coupling of $J_{K}=4$. As was the case for $a_{1}^{e} L$, the results for different system sizes again follow a single curve, validating the scaling $a_{1}^{o} \sim 1 / L^{2}$ predicted by theory. A term proportional to $1 / L$ can definitely be excluded for this Fourier coefficient. 


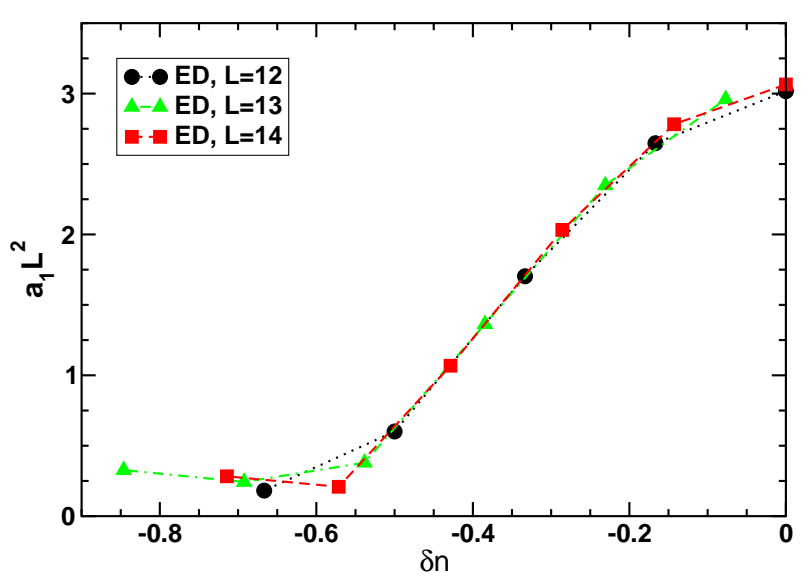

FIG. 4: The first Fourier coefficient scaled by $L^{2}, a_{1}^{o} L^{2}$, of the current for the SCQD away from $1 / 2$-filling, for $N$ odd as a function of $\delta n=(N-1) / L-1$. In all cases $J_{K}=4.0$. $\circ$ indicates ED results with $L=12$ and $N=13,11,9,7,5,3, \triangle$ indicates ED results with $L=13$ and $N=13,11,9,7,5,3$, finally - indicates ED results with $L=14$ and $N=15,13,11,9,7,5$. The lines are guides to the eye.

We also see that, for $\delta n \rightarrow 0$, our results are consistent with deviations from the result at half-filling being of higher than linear order in $\delta n$, although a definite conclusion is hard to obtain due to the limited system sizes available.

\section{FINITE TEMPERATURE CONDUCTANCE}

We now calculate the conductance for a quantum dot, side-coupled to infinitely long leads, at a low finite temperature, $T<<T_{K}$. We do this using our Fermi liquid Hamiltonian, of Eq. (2.18). We choose $L$ and $L / 2$ even, for convenience but we are now taking the $L \rightarrow \infty$ limit so this choice is immaterial. Working with left-movers only, and taking into account that the point $x=L$ corresponds to $x=0^{-}, x=0$ to $0^{+}$, we write the Fermi liquid interaction as:

$$
H_{\text {int }}=\frac{-8 \pi}{3 T_{K}}:\left[\psi^{\dagger} \frac{\vec{\sigma}}{2} \psi\right]^{2}:-\frac{4 i}{3 T_{K}}: \psi^{\dagger} \frac{\overleftrightarrow{d}}{d x} \psi:+ \text { const. }
$$

Here

$$
\psi \equiv \frac{1}{\sqrt{2}}\left[\psi_{L}\left(0^{+}\right)-\psi_{L}\left(0^{-}\right) e^{-i \alpha}\right],
$$

the : : denotes normal ordering and we define:

$$
f(x) \frac{\overleftrightarrow{d}}{d x} g(x) \equiv f \frac{d g}{d x}-\frac{d f}{d x} g
$$

The second, derivative term in Eq. (4.1), arises from a point splitting procedure. The second term is referred to as the elastic part of the interaction, corresponding to a single electron impuirity scattering process, while the first term is referred to as the inelastic part, corresonding to an electron-electron interaction at the origin. (For a derivation of the Fermi liquid interaction in this form, see [32], but note that the fermion fields are defined with an unconventional normalization there so that they are larger by a factor of $\sqrt{2 \pi}$.) Now the phase $\alpha$, is regarded as a time-dependent vector potential, related to the potential energy drop across the quantum dot by:

$$
d \alpha / d t=-\int_{0^{-}}^{0^{+}} d x E(x)=\Delta V .
$$

(In this section we set the electron charge, $e=1$, reinstating it at the end.) The conductance through a quantum dot, treating the leads as ballistic, is insensitive to where the electric field is applied so we have chosen, for convenience, to apply it right at the junction, over a region of width of order $\xi_{K}$.

We write the current operator as the rate of change of one-half the difference of the total number of electrons on the right hand side $(x>0)$ of the system minus the total number on the left hand side:

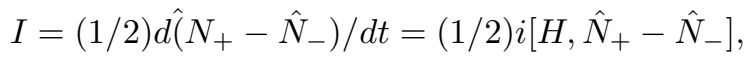

where

$$
\hat{N}_{+} \equiv \int_{0}^{\infty}\left[\psi_{L}^{\dagger}(x) \psi_{L}(x)+\psi_{R}^{\dagger}(x) \psi_{R}(x)\right],
$$

and similarly for $\hat{N}_{-}$. As discussed above, we can use the perfectly reflecting strong-coupling b.c. of Eqs. (2.4), (2.5) ) to rewrite the left and right movers at $x>0$ in terms of left-movers only on the entire real line. Thus we define:

$$
\begin{aligned}
\psi_{L+}(x) & \equiv \psi_{L}(x), \quad(x>0) \\
& =\psi_{R}(-x) \quad(x<0) .
\end{aligned}
$$

Here the subscript + indicates that this field derives from the $x>0$ region. Similarly, we define another left-moving field on the entire real line, $\psi_{L_{-}}(x)$ which derives from the region $x<0$ :

$$
\begin{aligned}
\psi_{L-}(x) & \equiv \psi_{L}(x), \quad(x<0) \\
& =\psi_{R}(-x) \quad(x>0) .
\end{aligned}
$$

In this notation:

$$
\hat{N}_{ \pm}=\int_{-\infty}^{\infty} \psi_{L \pm}^{\dagger}(x) \psi_{L \pm}(x)
$$

and the operator appearing in the Fermi liquid interaction, defined in Eq. (4.2) becomes:

$$
\psi \equiv \frac{1}{\sqrt{2}}\left[\psi_{L+}(0)-\psi_{L-}(0) e^{-i \alpha}\right] .
$$

We wish to calculate $\langle I\rangle$ in linear response to a time dependent vector potential $\alpha(t)$. At this point, there 
are two ways of proceeding. We may use the expression $I=(1 / 2) d(\Delta \hat{N}) / d t$ where

$$
\Delta \hat{N} \equiv \hat{N}_{+}-\hat{N}_{-},
$$

and ultimately relate the conductance to the Green's function of $\Delta \hat{N}$, or we may calculate explicitly the commutator $[H, \Delta \hat{N}]$ and relate the conductance to the Green's function of that operator. In both approaches the calculation must be carried out to second order in the Fermi liquid interaction and the amount of work is roughly the same either way. However, it is actually much more convenient to work with $d \Delta \hat{N} / d t$ because the needed Green's function can be related to the $\mathcal{T}$-matrix, which was calculated previously, ${ }^{10,32}$ This approach was used in 34] for instance, to calculate the conductance through an embedded quantum dot. The calculation is very similar in the side-coupled case and we may obtain the answer by only a slight modification of their result. This approach is used in the rest of this section. It is also of some interest to calculate the conductance using the commutator method and this is done in Appendix A, yielding precisely the same result.

In the $d \Delta \hat{N} / d t$ method, we use the fact that, to $O(\alpha)$, $H=H(0)+\alpha(t) I$, to obtain the conductance:

$$
C=\lim _{\omega \rightarrow 0} \frac{1}{\omega} \int_{0}^{\infty} d t e^{i \omega t}<[I(t), I(0)]>.
$$

Integrating by parts:

$$
C=(1 / 4) \lim _{\omega \rightarrow 0} \omega \int_{0}^{\infty} d \omega e^{i \omega t}<[\Delta \hat{N}(t), \Delta \hat{N}(0)]>
$$

where the retarded Green's function is evaluated at $\alpha=$ 0 . It is now convenient to go to the even-odd basis:

$$
\psi_{e / o}=\frac{1}{\sqrt{2}}\left(\psi_{L+} \mp \psi_{L_{-}}\right) .
$$

Since the interactions only involve $\psi_{e}, C$ factorizes into a free Green's function for $\psi_{o}$ multiplied by the non-trivial Green's function for $\psi_{e}$. This latter Green's function can be expressed in terms of the $\mathcal{T}$-matrix, giving a formula for the conductance: 36

$$
C=\frac{e^{2}}{h} \sum_{s} \int d \epsilon(-d f / d \epsilon)\left[-\pi \nu \operatorname{Im} \mathcal{T}_{s}(\epsilon)\right],
$$

where $s= \pm 1$ labels the fermion spin, $f(\epsilon)$ is the Fermi distribution function at temperature $T$ and $\nu$ is the density of states. As shown in [34] following [10, 32], for the embedded quantum dot:

$-\pi \nu T_{s}(\epsilon)=\frac{1}{2 i}\left[\exp \left[2 i \delta_{s}(\epsilon)\right]-1\right]+\exp \left[2 i \delta_{s}(\epsilon)\right]\left[-\pi \nu \tilde{\mathcal{T}}_{i n}(\epsilon)\right]$

where

$$
\begin{aligned}
\delta_{s}(\epsilon) & =s \pi / 2+\tilde{\delta}(\epsilon) \\
\tilde{\delta}(\epsilon) & =\omega / T_{K} \\
-\pi \nu \tilde{T}_{i n}(\epsilon) & =i \frac{\left(\epsilon^{2}+\pi^{2} T^{2}\right)}{2 T_{K}^{2}} .
\end{aligned}
$$

$\tilde{\delta}(\epsilon)$ and $\tilde{T}_{i n}(\epsilon)$ are the phase shift and inelastic part of the $\mathcal{T}$-matrix calculated in perturbation theory in the Fermi liquid interactions. The extra $s \pi / 2$ term in $\delta_{s}$ arises from the $\pm \pi / 2$ phase shift characterizing the strong coupling fixed point. This $\pm \pi / 2$ phase shift reflects the perfect transmission $\left(C=2 e^{2} / h\right)$ at $T=0$ for the embedded quantum dot. On the other hand, for the sidecoupled quantum dot, $C=0$ at the zero temperature fixed point, implying the absence of this extra phase shift. Thus, for the side-coupled quantum dot we have:

$$
-\pi \nu T_{s}(\epsilon)=\frac{1}{2 i}\left[\exp \left[2 i \tilde{\delta}_{s}(\epsilon)\right]-1\right]+\exp \left[2 i \tilde{\delta}_{s}(\epsilon)\right]\left[-\pi \nu \mathcal{T}_{i n}(\epsilon)\right]
$$

To order $\omega^{2}, T^{2}$, the difference between $\mathcal{T}$-matrices for the embedded and side-coupled quantum dots is just a change in sign in the first and third terms. This implies:

$$
\begin{aligned}
& -\pi \nu \operatorname{Im} \mathcal{T}(\epsilon)=1-\frac{\left(3 \epsilon^{2}+\pi^{2} T^{2}\right)}{2 T_{K}^{2}} \text { (embedded) } \\
& -\pi \nu \operatorname{Im} \mathcal{T}(\epsilon)=\frac{\left(3 \epsilon^{2}+\pi^{2} T^{2}\right)}{2 T_{K}^{2}} \quad \text { (side-coupled) }(4.19)
\end{aligned}
$$

Inserting these expressions into Eq. 4.15) for the conductance, and restoring $\hbar$ and $e$ which were previously set to one, gives:

$$
\begin{aligned}
C & =\frac{2 e^{2}}{h}\left[1-\frac{\pi^{2} T^{2}}{T_{K}^{2}}\right], \quad \text { (embedded) } \\
& =\frac{2 e^{2}}{h} \frac{\pi^{2} T^{2}}{T_{K}^{2}} \quad \text { (side-coupled) }
\end{aligned}
$$

We may now write universal Wilson-type ratios between the low temperature conductance, $C$, through a side-coupled quantum dot and the zero temperature persistent current, $j_{e}, j_{o}$, through the same quantum dot inserted into a ring of size $L \gg \xi_{K}$ :

$$
\begin{aligned}
& \frac{L^{2} j_{e} / e v_{F}^{2}}{\sqrt{h C /\left(2 e^{2} T^{2}\right)}} \rightarrow \frac{1}{4} \sin 2 \tilde{\alpha} \\
& \frac{L^{2} j_{o} / e v_{F}^{2}}{\sqrt{h C /\left(2 e^{2} T^{2}\right)}} \rightarrow \frac{1}{2} \sin \tilde{\alpha}-\frac{1}{4} \sin 2 \tilde{\alpha} .
\end{aligned}
$$

When PH symmetry is broken the conductance also gets a contribution of second order in $\lambda$ the coupling constant in the term, $\mathrm{H}_{2}$ of the effective Hamiltonian, given in Eq. (2.43). The simplest way of evaluating this contribution is to observe that, since the Hamiltonian is non-interacting, ignoring the other term $H_{\text {int }}$ in Eq. (2.18), we can evaluate the conductance at zero temperature using the Landauer formula, $C=\left(2 e^{2} / h\right) T_{r}$ where $T_{r}$ is the transmission probability through the quantum dot. For small $\lambda, T_{r}=\lambda^{2}$.

Including both terms, the conductance takes the form at low temperatures:

$$
C=\left[\frac{\pi^{2} T^{2}}{T_{K}^{2}}+\lambda^{2}\right] \frac{2 e^{2}}{h} .
$$


At sufficiently low $T$ the second, non-universal term, arising from PH symmetry breaking always dominates. However provided that the $\mathrm{PH}$ symmetry breaking (and, in particular, the direct tunnelling across the quantum dot) is small, the first, universal, term dominates for:

$$
\lambda T_{K}<<T<<T_{K} .
$$

Note that this situation is very analogous to that for the persistent current, with $T$ replaced by $v_{F} / L$.

\section{CONCLUSION}

We have developed a Fermi liquid theory of the persistent current in a side coupled quantum dot. This theory should correctly describe the limit $a \ll \xi_{K} \ll L$ where $a$ is a microscopic length scale. Numerical results are largely in agreement with the existence of a regime correctly described by this Fermi liquid picture. However, probably due to the limited system sizes available and the requirement that $L \gg \xi_{K}$, the numerical evidence cannot be described as strong. Our Fermi liquid theory confirms the existence of a term in the persistent current proportional to $\sin (\tilde{\alpha}) \delta n / J_{K}^{2} L$ for $N$ even, absent at halffilling. We have also calculated the conductance through a side-coupled quantum dot at low $T \ll T_{K}$ and calculated a universal "Wilson" ratio relating the conductance to the persistent current.

\section{APPENDIX A: CONDUCTANCE BY COMMUTATOR METHOD}

In this appendix we repeat the calculation of the conductance, writing the current as $I=i[H, \Delta \hat{N}] / 2$. This provides a check on the previous Fermi liquid calculations involving the $\mathcal{T}$-matrix in [10,32,34,36] and also provides an instructive example of how universal information can be extracted from a cut-off dependent result.

Since $\left[\hat{N}_{+}+\hat{N}_{-}, H\right]=0$, we may equivalently use $I=i\left[H, \hat{N}_{+}\right]$. Note that $\hat{N}_{+}$commutes with the noninteracting part of the Hamiltonian which is simply that of free fermions with perfectly reflecting b.c.'s at the origin, since this Hamiltonian does not transmit any electrons between left and right sides of the system. The commutator of $\hat{N}_{+}$with $H_{\text {int }}$ is readily calculated. Since all fields in the commutator are left-movers sitting at $x=0$, we drop the $L$ subscript and the (0) argument for simplicity. The result is:

$$
\begin{aligned}
I=i\left[H_{i n t}, N_{+}\right]= & -\frac{2 \pi i}{3 T_{K}}\left\{\left[e^{-i \alpha} \psi_{+}^{\dagger} \frac{\vec{\sigma}}{2} \psi_{-}-h . c .\right],\left[\psi_{+}-e^{-i \alpha} \psi_{-}\right]^{\dagger} \frac{\vec{\sigma}}{2}\left[\psi_{+}-e^{-i \alpha} \psi_{-}\right]\right\} \\
& +\frac{1}{2 T_{K}}\left[\psi_{+}^{\dagger} \frac{d}{d x} \psi_{-} e^{-i \alpha}-\frac{d}{d x} \psi_{+}^{\dagger} \psi_{-} e^{-i \alpha}+\text { h.c. }\right] .
\end{aligned}
$$

We note that this is, equivalently, $I=d H_{\text {int }} / d \alpha$. We expand the current operator up to first order in the vector potential, $\alpha$ :

$$
I=I_{0}+\alpha I_{1}+O\left(\alpha^{2}\right) .
$$

Here:

$$
\begin{aligned}
I_{0}= & \frac{4 \pi i}{3 T_{K}}\left[\left(\psi_{+}^{\dagger} \frac{\vec{\sigma}}{2} \psi_{-}\right)^{2}-\psi_{+}^{\dagger} \frac{\vec{\sigma}}{2} \psi_{-} \cdot\left(\vec{J}_{+}+\vec{J}_{-}\right)-\text {h.c. }\right] \\
& +\frac{1}{2 T_{K}}\left[\psi_{+}^{\dagger} \frac{\overleftrightarrow{d}}{d x} \psi_{-}+\text {h.c. }\right] \equiv I_{i n}+I_{e l},
\end{aligned}
$$

where

$$
\overrightarrow{J_{ \pm}} \equiv \psi_{ \pm}^{\dagger} \frac{\vec{\sigma}}{2} \psi_{ \pm}
$$

and we have defined the inelastic and elastic terms in the current operator which are, respectively, quartic and quadratic in fermion operators. The precise form of $I_{1}$ will not be needed. The conductivity is obtained from $<I>$, calculated to first order in $\alpha$. Thus we obtain:

$$
I(\omega)=\left[G_{R}(\omega)+<I_{1}>\right] \alpha(\omega),
$$

where $G_{R}$ is the retarded Green's function:

$$
G_{R}(\omega)=-i \int_{0}^{\infty} e^{i \omega t}<\left[I_{0}(t), I_{0}(0)\right]>_{T} .
$$

It can be seen that $I=0$ when $\alpha(t)$ is a constant, independent of $t$. A non-zero $I$ in this case would correspond to a persistent current, but this must vanish for infinite $L$ since a constant phase $\alpha$ in $H_{\text {int }}$ can be eliminated by a gauge transformation in that limit. Thus we conclude that $\left\langle I_{1}\right\rangle=-G_{R}(0)$, so that:

$$
I(\omega)=\left[G_{R}(\omega)-G_{R}(0)\right] \alpha(\omega) .
$$

From Eq. (4.4), we see that:

$$
I(\omega)=C(\omega) \Delta V(\omega)
$$

where the conductance, $C(\omega)$, is given by:

$$
C(\omega)=\frac{i}{\omega}\left[G_{R}(\omega)-G_{R}(0)\right],
$$


where $\omega_{n}=2 \pi n T$ and $\beta \equiv 1 / T$. (We set Boltzmann's constant equal to one.) The dc conductance is:

$$
C=\lim _{\omega \rightarrow 0} \frac{i}{\omega}\left[G_{R}(\omega)-G_{R}(0)\right] .
$$

We calculate the retarded Green's function of $I_{0}$ by analytic continuation from the imaginary time, Matsubara Green's function:

$$
\mathcal{G}\left(i \omega_{n}\right)=-\int_{0}^{\beta} d \tau e^{i \omega_{n} \tau}<I_{0}(\tau) I_{0}(0)>.
$$

The free fermion Matsubara Green's function, at late times, using the same normalization as in Eq. (2.22), that was used in the calculation of the persistent current is:

$$
<\psi_{i}^{\dagger \alpha}(\tau) \psi_{\gamma j}(0)>\rightarrow \frac{\delta_{\gamma}^{\alpha} \delta_{i j}}{2 \beta \sin (\pi \tau / \beta)}
$$

where $i, j= \pm$ labels right and left sides of the junction, $x=0^{ \pm}$and $\beta$ is the inverse temperature. Using Wick's theorem, the identity:

$$
\sum_{a, b}\left[\operatorname{tr} \sigma^{a} \sigma^{b} \operatorname{tr} \sigma^{a} \sigma^{b}-\operatorname{tr} \sigma^{a} \sigma^{b} \sigma^{a} \sigma^{b}\right]=18,
$$

and collecting the various terms, we obtain the long-time behavior of the Matsubara Green's function:

$$
<I_{\text {in }}(\tau) I_{\text {in }}(0)>\rightarrow \frac{16 \pi^{2}}{T_{K}^{2}} \frac{1}{(2 \beta)^{4} \sin ^{4}(\pi \tau / \beta)} .
$$

To evaluate $<I_{e l}(\tau) I_{e l}(0)>$, we use the derivatives of the free fermion Green's function:

$$
\begin{aligned}
\frac{d}{d x}\left\{\frac{1}{\sin [\pi(\tau+i x) / \beta]}\right\} & =-\frac{i \pi \cos [\pi(\tau+i x) / \beta]}{\beta \sin ^{2}[\pi(\tau+i x) / \beta]}, \\
\frac{d^{2}}{d x^{2}} \frac{1}{\sin [\pi(\tau+i x) / \beta]} & =-\frac{\pi^{2}}{\beta^{2}} \frac{1+\cos ^{2}[\pi(\tau+i x) / \beta]}{\sin ^{3}[\pi(\tau+i x) / \beta]} .
\end{aligned}
$$

The terms proportional to $\cos ^{2}(\pi \tau / \beta)$ cancel leaving:

$$
\begin{aligned}
<I_{e l}(\tau) I_{e l}(0)> & \rightarrow \frac{8 \pi^{2}}{T_{K}^{2}} \frac{1}{(2 \beta)^{4} \sin ^{4}(\pi \tau / \beta)} \\
& =(1 / 2)<I_{i n}(\tau) I_{\text {in }}(0)>
\end{aligned}
$$

We now consider the Fourier transform of $G(\tau)$ at low frequencies and temperatures. The analytic continuation of $\mathcal{G}\left(i \omega_{n}\right)\left(\omega_{n} \equiv 2 \pi n / \beta\right)$ to real frequencies gives the retarded Green's function:

$$
\mathcal{G}\left(i \omega_{n} \rightarrow \omega+i \delta\right)=G_{R}(\omega)
$$

where $\delta$ is a postive infinitesimal. We will find that

$$
\mathcal{G}\left(i \omega_{n}\right) \rightarrow A+B\left|\omega_{n}\right|+O\left(\omega_{n}^{2}\right),
$$

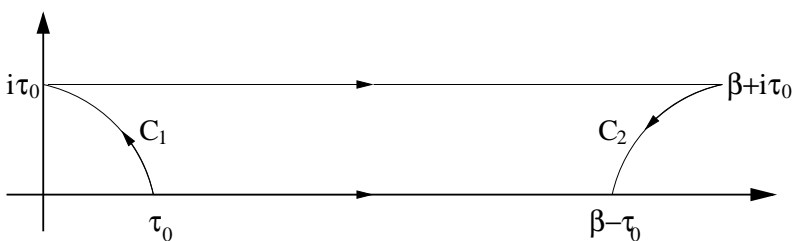

FIG. 5: Contour used to evaluate the integral in Eq. A21.

for $\omega_{n}<<D$. The analytic continuation gives:

$$
\begin{aligned}
\left|\omega_{n}\right| & =\omega_{n} \int_{-\infty}^{\infty} \frac{d \tau}{2 \pi i} \frac{e^{i \omega_{n} \tau}-e^{-i \omega_{n} \tau}}{\tau-i \epsilon} \\
& \rightarrow(-i \omega) \int_{-\infty}^{\infty} \frac{d \tau}{2 \pi i} \frac{e^{(i \delta-\omega) \tau}-e^{-i(\delta-i \omega) \tau}}{\tau-i \epsilon} \\
& =-i \omega .
\end{aligned}
$$

$G\left(\omega_{n}\right)$, which is an even function of $\omega_{n}$, in our assumed particle-hole symmetric model, contains non-universal ultraviolet cut-off dependent terms. Introducing a cut-off, $\tau_{0}$ with dimensions of time, and of order $1 / D$, we find, at low $\omega_{n}$ :

$$
\begin{aligned}
& \mathcal{G}\left(i \omega_{n}\right) \rightarrow \\
& \quad \frac{1}{T_{K}^{2}}\left[\frac{a}{\tau_{0}^{3}}+b \frac{1}{\tau_{0} \beta^{2}}+c \frac{\left|\omega_{n}\right|}{\beta^{2}}+d \frac{\omega_{n}^{2}}{\tau_{0}}+e\left|\omega_{n}\right|^{3}+\ldots\right],
\end{aligned}
$$

where $a, b, c, d, e$ are dimensionless numbers. While the terms of $\mathrm{O}\left(\omega_{n}^{0}\right)$ and $\left(\omega_{n}^{2}\right)$ are non-universal and cut-off dependent, we expect that the terms $\propto\left|\omega_{n}\right|$ and $\left|\omega_{n}\right|^{3}$ are not. This is related to the fact that only these terms are singular functions of $\omega_{n}$. This singularity arises from the universal long-time behavior of $G(\tau)$. Note that the dc conductance is completely determined by the universal $c$-term $\propto\left|\omega_{n}\right|$ and so is a universal quantity in the sense that it is independent of the details of the ultraviolet cut-off. To verify these assertions, we consider a particularly simple ultraviolet cut-off. The $\tau$ integral defining the Fourier transform of the current Green's function is restricted to $\tau_{0}<\tau<\beta-\tau_{0}$ Thus we begin with:

$$
\mathcal{G}\left(i \omega_{n}\right) \equiv \frac{-24 \pi^{2}}{T_{K}^{2}(2 \beta)^{4}} \int_{\tau_{0}}^{\beta-\tau_{0}} \frac{d \tau e^{i \omega_{n} \tau}}{\sin ^{4}(\pi \tau / \beta)} .
$$

This integral can be straightforwardly developed in an expansion in $\tau_{0} / \beta$, which has the form of Eq. A20). This can be conveniently done by deforming the $\tau$-integral into the complex plane. The original integral is equal to the sum of an integral along the straight line from $\tau=i \tau_{0}$ to $\tau=i \tau_{0}+\beta$ plus the integral on two quarter-circle contours from $\tau=\tau_{0}$ to $\tau=i \tau_{0}$ and from $\beta+i \tau_{0}$ to $\tau=\beta-\tau_{0}$, which we label $C_{1}$ and $C_{2}$ respectively. [See fig. (5).] The integral along the straight line can be written:

$$
K=\int_{0}^{\beta} \frac{d \tau e^{i \omega_{n}\left(\tau+i \tau_{0}\right)}}{\sin ^{4}\left[\pi\left(\tau+i \tau_{0}\right) / \beta\right]} .
$$


We may now Taylor expand the denominator:

$\frac{1}{\sin ^{4}\left[\pi\left(\tau+i \tau_{0}\right) / \beta\right]}=16 e^{4 \pi i\left(\tau+i \tau_{0}\right) / \beta} \sum_{m=0}^{\infty} a_{m} e^{2 \pi i m\left(\tau+i \tau_{0}\right) / \beta}$.

Note that the sum converges due to the factors $e^{-2 \pi \tau_{0} / \beta}$. Here the $a_{m}$ are the binomial coefficients:

$$
a_{m}=(m+1)(m+2)(m+3) / 6 .
$$

We now integrate over $\beta$ term by term. The result is:

$$
\int_{0}^{\beta} d \tau e^{i\left[\omega_{n}+(2+m)(2 \pi / \beta)\right] \tau}=\beta \delta_{n,-(2+m)} .
$$

(Recall that $\omega_{n} \equiv 2 \pi n / \beta$.) Thus we obtain:

$$
K=-\frac{4 \beta^{2}\left|\omega_{n}\right|}{3 \pi}\left[1-\left(\frac{\beta \omega_{n}}{2 \pi}\right)^{2}\right] \theta\left(-\omega_{n}\right) .
$$

Alternatively, the integral of Eq. A22 can be evaluated by introducing the complex variable $z \equiv e^{i 2 \pi\left(\tau+i \tau_{0}\right) / \beta}$.
The integration contour for $z$ is a circle of radius $e^{-2 \pi \tau_{0} / \beta}<1$. This contour encloses a pole of order $|n|-1$ when $n<-1$.

We also must perform the integration over the two quarter-circle contours:

$$
K_{12} \equiv \sum_{i=1}^{2} \int_{C_{i}} d z \frac{e^{i \omega_{n} z}}{\sin ^{4}(\pi z / \beta)}
$$

On $C_{1}$ we write $z \equiv \tau_{0} e^{i \theta}$ where $\theta$ goes from $0 \rightarrow \pi / 2$ along the contour. On $C_{2}$ we write $z=\beta+\tau_{0} e^{i \theta}$ where now $\theta$ goes from $\pi / 2 \rightarrow \pi$. Since the integrand is invariant under a translation: $z \rightarrow z+\beta$, we may combine these two terms to write:

$$
K_{12}=i \tau_{0} \int_{0}^{\pi} d \theta \frac{e^{i \theta} \exp \left[i \omega_{n} \tau_{0} e^{i \theta}\right]}{\sin ^{4}\left(\pi \tau_{0} e^{i \theta} / \beta\right)}
$$

Next we expand $\exp \left[i \omega_{n} \tau_{0} e^{i \theta}\right]$ in powers of $\omega_{n} \tau_{0}$ and expand $\sin ^{-4}\left(\pi \tau_{0} e^{i \theta} / \beta\right)$ in powers of $\tau_{0} / \beta$, giving:

$$
K_{12}=i \tau_{0}\left(\frac{\beta}{\pi \tau_{0}}\right)^{4} \int_{0}^{\pi} e^{i \theta} e^{-4 i \theta}\left[1+i \omega_{n} \tau_{0} e^{i \theta}-\frac{\omega_{n}^{2} \tau^{2}}{2} e^{2 i \theta}-\frac{i \omega_{n}^{3} \tau_{0}^{3}}{6} e^{3 i \theta}+\ldots\right]\left[1-\frac{\pi^{2} \tau_{0}^{2}}{6 \beta^{2}} e^{2 i \theta}+\frac{\pi^{4} \tau_{0}^{4}}{120 \beta^{4}} e^{4 i \theta}+\ldots\right]^{-4}
$$

We Taylor expand, collect terms, and integrate term by term using:

$$
\begin{aligned}
\int_{0}^{\pi} d \theta e^{i m \theta} & =\pi \quad(m=0) \\
& =0 \quad(m \text { even }, \quad m \geq 2) \\
& =2 i / m, \quad(m \text { odd })
\end{aligned}
$$

Note that non-zero terms from the expansion must be proportional to $e^{3 i \theta}$ or else $e^{2 i m \theta}$ to give a non-zero contribution. The first type of terms are odd functions of $\omega_{n}$, proportional to $\omega_{n}$ and $\omega_{n}^{3}$. All other terms are even functions of $\omega_{n}$. Keeping only the terms which are nonvanishing as $\tau_{0} \rightarrow 0$ gives:

$K_{12} \approx \frac{-2 \omega_{n} \beta^{2}}{3 \pi}+\frac{\omega_{n}^{3} \beta^{4}}{6 \pi^{3}}+\frac{2 \beta^{4}}{3 \pi^{4} \tau_{0}^{3}}-\frac{\omega_{n}^{2} \beta^{4}}{\pi^{4} \tau_{0}}+\frac{4 \beta^{2}}{3 \pi^{2} \tau_{0}}+\ldots$

The omitted terms are all even functions of $\omega_{n}$ and vanish as $\tau_{0} \rightarrow 0$.

Note that when we add $K+K_{12}$ we regain an even function of $\omega_{n}$, as we must:

$$
\begin{aligned}
K+K_{12} & =\frac{-2 \beta^{2}}{3 \pi}\left|\omega_{n}\right|\left[1-\left(\frac{\beta \omega_{n}}{2 \pi}\right)^{2}\right] \\
& +\frac{2 \beta^{4}}{3 \pi^{4} \tau_{0}^{3}}-\frac{\omega_{n}^{2} \beta^{4}}{\pi^{4} \tau_{0}}+\frac{4 \beta^{2}}{3 \pi^{2} \tau_{0}}+\ldots
\end{aligned}
$$

The first two terms are singular functions of $\omega_{n}$ at $\omega_{n}=0$. All remaining terms are non-singular, even powers of $\omega_{n}$. The singular terms are cut-off independent, unlike the non-singular ones.

Thus we may write the imaginary frequency Green's function for the current operator as:

$$
\mathcal{G}\left(i \omega_{n}\right) \rightarrow \mathcal{G}(0)+\frac{\pi T^{2}\left|\omega_{n}\right|}{T_{K}^{2}}+O\left(\omega_{n}^{2}\right)
$$

The analytic continuation to real frequency is straightforward, using Eq. (A19):

$$
G_{R}(\omega) \rightarrow G_{R}(0)-\frac{i \pi T^{2} \omega}{T_{K}^{2}}+O\left(\omega^{2}\right)
$$

Thus, we obtain the dc conductance from Eq. (A10):

$$
C=\frac{\pi T^{2}}{T_{K}^{2}} \rightarrow \frac{2 e^{2}}{h} \frac{\pi^{2} T^{2}}{T_{K}^{2}},
$$

the same result obtained from the derivative method, Eq. (4.20). (A factor of $e^{2} / \hbar$, previously set equal to one was inserted in the last step.)

In Sec. V, we evaluated the conductance from the P-H symmetry violating tunneling term using the Landauer formalism. It is instructive to evaluate the conductance instead using the Kubo formula, and the commutator 
approach, as done earlier in this Appendix. In this case, the new term in the $(\alpha=0)$ current operator is:

$$
\delta I_{0}=i \lambda e^{i \pi(N-1) / 2} \psi_{+}^{\dagger} \psi_{-}+\text {h.c. }
$$

The Green's function for $I_{0}$, for large imaginary times, now picks up a correction:

$$
\delta<I_{0}(\tau) I_{0}(0)>=4 \lambda^{2} \frac{1}{(2 \beta)^{2} \sin ^{2}(\pi \tau / \beta)} .
$$

We may evaluate the Fourier transform using the same methods as above. However, we focus immediately on the $T=0$ limit, for simplicity:

$$
\delta \mathcal{G}\left(\omega_{n}\right)=-4 \lambda^{2} \int_{-\infty}^{\infty} \frac{d \tau e^{i \omega_{n} \tau}}{(2 \pi \tau)^{2}} .
$$

This integral requires an ultraviolet cut off, as before. Cutting off the integral at $|\tau|>\tau_{0}$, gives:

$$
\delta \mathcal{G}=\frac{-2 \lambda^{2}}{\pi^{2}} \int_{\tau_{0}}^{\infty} \frac{d \tau}{\tau^{2}} \cos \left(\omega_{n} \tau\right)
$$

It is convenient to integrate by parts, giving:

$$
\delta \mathcal{G}=\frac{-2 \lambda^{2}}{\pi^{2} \tau_{0}}+\frac{2 \lambda^{2} \omega_{n}}{\pi^{2}} \int_{\tau_{0}}^{\infty} \frac{d \tau}{\tau} \sin \left(\omega_{n} \tau\right) .
$$

[We used $\cos \left(\omega_{n} \tau_{0}\right) \approx 1$ in the first term.] Since this integral now converges, we may take $\tau_{0} \rightarrow 0$, assuming that $\left|\omega_{n}\right| \tau_{0} \ll 1$. We may then rescale the integral giving:

$$
\delta \mathcal{G}=\frac{-2 \lambda^{2}}{\pi^{2} \tau_{0}}+\frac{2 \lambda^{2}\left|\omega_{n}\right|}{\pi^{2}} \int_{0}^{\infty} \frac{d s}{s} \sin s+O\left(\tau_{0}\right) .
$$

Evaluating the integral gives:

$$
\delta \mathcal{G}=\frac{-2 \lambda^{2}}{\pi^{2} \tau_{0}}+\frac{2 \lambda^{2}\left|\omega_{n}\right|}{2 \pi}+O\left(\tau_{0}\right) .
$$

Note that we again obtain a term which is even in $\omega_{n}$ but singular, involving an absolute value. Analytically continuing to real frequency, using Eq. A19) we obtain the zero temperature DC conductance from Eq. (A10):

$$
C=\frac{\lambda^{2}}{\pi} \rightarrow \frac{2 e^{2}}{h} \lambda^{2}
$$

the same result than is obtained from the Landauer formula. (Again a factor of $e^{2} / \hbar$ was inserted at the last step.)

Alternatively, we could cut off the integral in Eq. (A38), by a finite bandwidth, $2 D$. Then the fermion propogator is modified to:

$$
<\psi_{i}^{\dagger \alpha}(\tau) \psi_{\beta j}(0)>\rightarrow \delta_{\beta}^{\alpha} \delta_{i j} \frac{1-e^{-D|\tau|}}{2 \pi \tau},
$$

giving:

$$
\delta G\left(\omega_{n}\right)=4 \lambda^{2} \int_{-\infty}^{\infty} \frac{d \tau e^{i \omega_{n} \tau}\left(1-e^{-D|\tau|}\right)^{2}}{(2 \pi \tau)^{2}}
$$

where the integration region is now the entire real line. This can be expressed in terms of the exponential integral function, $E_{i}(x)$, giving a term of $O(D)$, the same universal term, $-\lambda^{2}\left|\omega_{n}\right| / \pi$, plus terms that vanish when $1 / D \rightarrow 0$. This confirms that the term $\propto\left|\omega_{n}\right|$, which determines the conductance, is indeed universal.

\section{APPENDIX B: DEFINITIONS OF $T_{K}$}

The definition of $T_{K}$ used in this paper, and defined in Eq. (2.16), is the one first introduced by Nozières $\frac{10}{10}$ in the original paper on local Fermi Liquid Theory for the Kondo model. It corresponds to an arbitrary choice but does have the advantage that the coefficient of $T^{2} / T_{K}^{2}$ in the conductance has the relatively simple value of $\pi^{2}$. [See Eq. (4.20).] Another popular definition is the one adopted by Wilson, which we refer to as $T_{K}^{W}$. This is fixed by the requirement that the impurity susceptibility, at $T \gg T_{K}$ have a particular form:

$$
\begin{aligned}
& \chi_{i m p} \rightarrow \\
& \qquad \frac{\left(g \mu_{B}\right)^{2}}{4 T}\left[1-\frac{1}{\ln \left(T / T_{K}^{W}\right)}-\frac{(1 / 2) \ln \left[\ln \left(T / T_{K}^{W}\right)\right]}{\ln ^{2}\left(T / T_{K}^{W}\right)}\right. \\
& \left.\quad+O\left[\ln ^{2}\left[\ln \left(T / T_{K}^{W}\right)\right] / \ln ^{3}\left(T / T_{K}^{W}\right)\right]\right] .
\end{aligned}
$$

At low temperatures,

$$
\chi_{i m p} \rightarrow \frac{\left(g \mu_{B}\right)^{2} w^{2}}{4 T_{K}^{W}},
$$

where the Wilson number, $w$ has the value:

$$
w=e^{C+1 / 4} / \pi^{3 / 2} \approx .4128
$$

and $C$ is Euler's constant $(\approx .577216)$. These definitions of $T_{K}$ are related by:

$$
T_{K}^{W}=(\pi w / 4) T_{K} .
$$

Rather accurate results have been determined for the conductance at all temperatures using numerical renormalization group 37 and integrability $\underline{38}$ These authors generally use a Kondo temperature, $T_{K}^{C}$, related to the other ones by:

$$
T_{K}^{C}=T_{K}^{W} / w=(\pi / 4) T_{K},
$$

motivated, perhaps, by the fact that the zero temperature impurity susceptibility now takes the simple form $\chi_{i m p} \rightarrow\left(g \mu_{B}\right)^{2} /\left(4 T_{K}^{C}\right)$. Some experimental papers ${ }^{2.4}$ on the Kondo effect in embedded quantum dots define a Kondo temperature by the condition that the conductance take half its zero temperature value at $T=T_{K}^{e}$ :

$$
C\left(T=T_{K}^{e}\right) / C(T=0)=1 / 2 .
$$


The theoretical results in [38] indicate that $T_{K}^{e} \approx T_{K}^{C}$. In [32] we wrote the Fermi liquid interaction:

$$
H_{i n t}=-\lambda\left(\psi_{e}^{\dagger} \frac{\vec{\sigma}}{2} \psi_{e}\right)^{2}
$$

(where $\lambda$ is not to be confused with the coefficient of the particle-hole breaking term in the current paper) and we normalized our fermions in an unconventional way so that $\psi_{A L}=\sqrt{2 \pi} \psi$. Thus:

$$
\lambda=\frac{2}{3 \pi T_{K}} .
$$

Numerous other definitions of $T_{K}$ are also in use including, for example, the frequency scale at which $\operatorname{Im} \mathcal{T}(\omega, T=0)$ is reduced by $1 / 2$ from its maximum at zero frequency.

\section{ACKNOWLEDGMENTS}

This research is supported by NSERC, CFI, SHARCNET and CIAR. IA acknowledges interesting conversations with P. Simon.
1 D. Goldhaber-Gordon, H. Shtrikman, D. Mahalu, D. Abusch-Magder, U. Meirav, and M. A. Kastner, Nature 391, 156 (1998).

2 D. Goldhaber-Gordon, J. Gores, M. A. Kastner, H. Shtrikman, D. Mahalu, and U. Meirav, Phys. Rev. Lett. 81, 5225 (1998).

3 S. M. Cronenwett, T. H. Oosterkamp, and L. P. Kouwenhoven, Science 281, 540 (1998).

${ }^{4}$ W. G. van der Wiel and et. al, Science 289, 2105 (2000).

${ }^{5}$ H. C. Manoharan and et. al, Nature 403, 512 (2000).

6 J. Park and et. al, Nature 417, 722 (2002).

7 W. Liang, M. P. Shores, M. Bockrath, J. R. Long, and H. Park, Nature 417, 725 (2002).

8 J. Nygård, D. H. Cobden, and P. E. Lindelof, Nature 408, $342(2000)$.

9 L. P. Kouwenhoven and L. I. Glazman, Phys. World 14, 33 (2001).

10 P. Nozières, J. Low Temp. Phys. 17, 31 (1974).

11 E. S. Sørensen and I. Affleck, Phys. Rev. B 53, 9153 (1996).

12 V. Barzykin and I. Affleck, Phys. Rev. Lett. 76, 4959 (1996).

13 V. Barzykin and I. Affleck, Phys. Rev. B 57, 432 (1998).

14 P. S. Cornaglia and C. A. Balseiro, Phys. Rev. Lett. 90, 216801 (2003).

15 Torio, K. Hallberg, and C. Proetto (2004), condmat/0404146.

16 L. Borda (2006), cond-mat/0611208.

17 W. B. Thimm, J. Kroha, and J. von Delft, Phys. Rev. Lett. 82, 2143 (1999).

18 T. Hand, J. Kroha, and H. Monien, Phys. Rev. Lett. 97, 136604 (2006).

19 A. A. Zvyagin and T. V. Bandos, Soviet Journal of Low. Temp. Phys. 20, 222 (1994).
20 A. A. Zvyagin and P. Schlottmann, Phys. Rev. B 54, 15191 (1996)

21 V. Ferrari, G. Chiappe, E. V. Anda, , and M. A. Davidovich, Phys. Rev. Lett. 82, 5088 (1999).

${ }^{22}$ K. Kang and S.-C. Shin, Phys. Rev. Lett. 85, 5619 (2000).

23 S. Y. Cho, K. Kang, C. K. Kim, and C.-M. Ryu, Phys. Rev. B 64, 033314 (2001).

${ }^{24}$ H.-P. Eckle, H. Johannesson, and C. A. Stafford, Phys. Rev. Lett. 87, 016602 (2001).

25 I. Affleck and P. Simon, Phys. Rev. Lett. 86, 2854 (2001).

26 P. Simon and I. Affleck, Phys. Rev. B 64, 085308 (2001).

27 A. A. Zvyagin, Phys. Rev. Lett. 87, 179704 (2001).

28 H. Hu, G.-M. Zhang, and L. Yu, Phys. Rev. Lett. 86, 5558 (2001).

29 A. A. Aligia, Phys. Rev. B 66, 165303 (2002).

30 E. S. Sørensen and I. Affleck, Phys. Rev. Lett. 94, 086601 (2005).

31 K. G. Wilson, Rev. Mod. Phys. 47, 773 (1975).

32 I. Affleck and A. W. W. Ludwig, Phys. Rev. B 48, 7297 (1993).

33 R. Shankar, Rev. Mod. Phys. 66, 129 (1994).

34 L. Glazman and M. Pustilnik, in Nanophysics: Coherence and Transport, edited by $\mathrm{H}$. Bouchiat and et al. (Elsevier, 2005), pp. 427-478.

35 A. O. Gogolin and N. V. Prokof'ev, Phys. Rev. B 50, 4921 (1994).

36 M. Pustilnik and L. I. Glazman, Phys. Rev. B 64, 045328 (2001).

37 T. A. Costi, A. C. Hewson, and V. Zlatic, J. Phys. Cond. Matt. 6, 2519 (1994).

38 R. M. Konik, H. Saleur, and A. Ludwig, Phys. Rev. B 66, 125304 (2002). 